\author{
G. 0. Antoine \\ Department of Biomedical Engineering and \\ Mechanics, \\ $\mathrm{M} / \mathrm{C} 0219$ \\ Virginia Polytechnic Institute and \\ State University, \\ Blacksburg, VA 24061 \\ e-mail: antoineg@vt.edu \\ R. C. Batra ${ }^{1}$ \\ Fellow ASME \\ Department of Biomedical Engineering and \\ Mechanics, \\ $\mathrm{M} / \mathrm{C}$ 0219, \\ Virginia Polytechnic Institute and \\ State University, \\ Blacksburg, VA 24061 \\ e-mail: rbatra@vt.edu
}

\section{Effect of Curvature on \\ Penetration Resistance of Polycarbonate Panels}

Three-dimensional transient deformations of clamped flat and doubly curved polycarbonate $(P C)$ panels impacted by a rigid smooth hemispherical-nosed circular cylinder have been numerically studied by the finite-element $(F E)$ method to delineate effects of the panel radius of curvature to its thickness ratio on their penetration resistance. The $P C$ is modeled as thermoelastoviscoplastic with the effective plastic strain rate depending upon the hydrostatic pressure. The effective plastic strain of 3.0 at failure is ascertained by matching for one set of flat panels the computed and the experimental minimum perforation speeds. It is found that a negative curvature (i.e., the center of curvature toward the impactor) of a panel degrades its penetration performance, and the positive curvature enhances it especially for thin panels with thickness/radius of curvature of 0.01. However, the benefit is less evident for panels with the panel thickness/radius of curvature of 0.04 or more. For positively curved thin panels, an elastic hinge forms around the central impacted area during an early stage of deformations, and subsequent deformations occur within this region. No such hinge is observed for flat plates, negatively curved panels of all the thicknesses, and positively curved thick panels. Furthermore, the maximum effective stress induced in regions surrounding the impacted area is less for positively curved panels than that for flat panels. The dominant failure mechanism is found to be the deletion of failed elements due to the effective plastic strain in them exceeding 3.0 rather than due to plug formation. For an example problem, the dependence of the effective plastic strain rate upon the hydrostatic pressure and the consideration of the Coulomb friction at the contact surfaces exhibited minimal effects on the penetration characteristics. This information should be useful for designers of impact-resistant transparent armor, such as an airplane canopy, automobile windshield, and goggles.

[DOI: $10.1115 / 1.4034520]$

Keywords: impact, curved panels, thermoelastoviscoplastic material, ductile failure

\section{Introduction}

Polymers are widely used as transparent armor because of their high-specific impact performance, e.g., see Radin and Goldsmith [1]. Sands et al. [2] reported that polycarbonates (PCs) have better specific impact resistance than most glasses. Thus, lightweight transparent PC panels are well-suited for protection in applications, such as goggles, shields, and windows. Mathematical and computational models that accurately predict the response of these panels to impact loads will help optimize their design for a given areal density.

Deformations of PC have been experimentally investigated by many researchers [3-11]. Test results show that after deforming elastically, the PC material exhibits plastic yielding followed by strain softening and finally strain hardening. An increase in temperature decreases both Young's modulus and the yield stress while increasing the strain rate has the same effect as decreasing the temperature. Moreover, there is no yield surface assumed, plastic deformations occur for all values of the stress, and the effective plastic strain rate depends upon the hydrostatic pressure.

The energy dissipated during plastic deformations of the material is generally converted into heat [12-14]. Assuming locally adiabatic heating of PC samples deformed at high strain rates, Rittel [14] introduced two parameters $\beta_{\text {diff }}$ and $\beta_{\text {int }}$ relating the rate of heating to the plastic working and the accumulated heating to the total plastic work, respectively. He found that for strain rates greater than $5000 / \mathrm{s}, \beta_{\text {int }}$ varies between 0.4 and 1 , and $\beta_{\text {diff }}$ can take values larger than the intuitively maximum value of 1.0

\footnotetext{
${ }^{1}$ Corresponding author

Manuscript received May 31, 2016; final manuscript received August 22, 2016 published online September 13, 2016. Assoc. Editor: Weinong Chen.
}

because of the likely conversion of the elastic energy into heat during the strain softening regime of deformations.

Several constitutive relations are available in the literature to model the thermomechanical response of a PC [5,15-17]. These are usually developed by decomposing the material response into a nonlinear elastic contribution accounting for the restoring force and a viscoelastoplastic contribution accounting for the strain-rate dependent plastic deformations (yielding, softening, and strain hardening). Here, we employ Mulliken and Boyce's [5] constitutive relation with Varghese and Batra's $[18,19]$ modifications of material parameters depending upon the current rather than the temperature in the unstressed reference configuration. This material model can be represented as a nonlinear Langevin spring and two nonlinear spring-dashpots in parallel to simulate, respectively, the restoring force and the viscoplastic response of the material. It includes the dependence of the effective plastic strain rate upon the pressure, the temperature, and the stress and strain rates. Safari et al.'s [20] extension of the material model to strain rates $>10^{4} / \mathrm{s}$ is not considered here.

Many experimental investigations on the fracture of PCs have used the Charpy tests and the three-point bend tests on notched specimens, e.g., see Refs. [21-28]. Craze nucleation has been identified as a principal failure mechanism of PCs at high strain rates [10,29-31]. Gunnarsson et al. [31] experimentally observed that flat PC panels impacted at $\sim 90 \mathrm{~m} / \mathrm{s}$ did not exhibit cracking and only showed a "small tear." Thus, only ductile failure of the $\mathrm{PC}$ is considered here.

Various damage and ductile failure models [11,32-38] used in numerical simulations of PCs are summarized in Table 1 .

Most failure criteria listed in Table 1 assume that a material point fails when the effective plastic strain there reaches a preassigned critical value. In Ref. [33], the critical value for the 
Table 1 Failure criteria and values of the failure parameters of PC used in numerical simulations

\begin{tabular}{lllr}
\hline \hline Reference & Code & \multicolumn{1}{c}{ Failure criterion } & Typical impact velocity (m/s) \\
\hline Ramakrishnan [11] & LS-DYNA & Critical plastic strain =0.5 & 4 \\
Kelly [32] & AUTODYN & Johnson-Holmquist failure and damage & 500 \\
Dorogoy et al. [33] & ABAQUS & Spallation at $p_{\text {crit }}=-160 \mathrm{MPa}$ & 750 \\
& & Damage initiation at plastic strain between 0.6 and 1.0; & \\
& & failure at 80 $\mu$ m plastic displacement & 140 \\
Shah and Abakr [34] & LS-DYNA & Critical plastic strain = 1.5 & 100 \\
Shah [35] & LS-DYNA & Critical plastic strain = 1.5 & 700 \\
Livingstone et al. [36] & AUTODYN & Bulk-strain; value not given by authors & 700 \\
Richards et al. [37] & AUTODYN & Bulk-strain; value not given by authors & 800 \\
Hazell et al. [38] & AUTODYN & Critical plastic strain = 2 & \\
\hline \hline
\end{tabular}

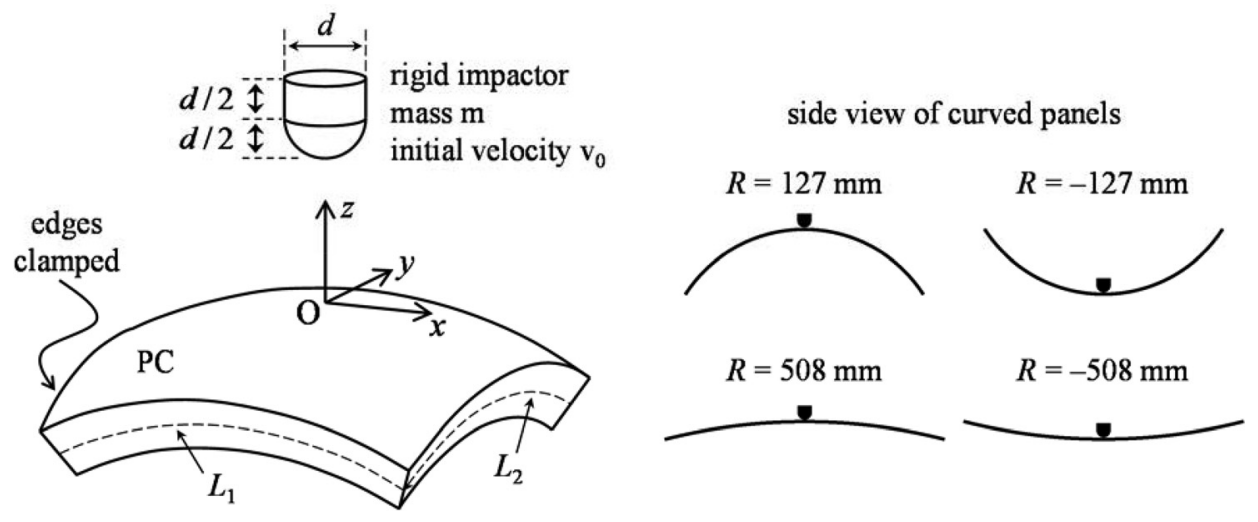

Fig. 1 Sketch of the impact problem studied

plastic strain at the onset of ductile damage is assumed to decrease from 1.0 for quasi-static deformations to 0.6 at the effective strain rate of $80,000 / \mathrm{s}$. Subsequently, elastic properties of the material are degraded, and the material is assumed to fail when the plastic displacement there equals $80 \mu \mathrm{m}$. For the finite-element (FE) mesh they used this plastic displacement corresponds to 0.064 additional plastic strain after the damage onset.

We note that the critical strain for the PC considered by various investigators varies from 0.66 [33] to 2 [38]. Thus, there is no agreement on the value of the critical plastic strain at failure. Here, we find the value 3.0 at failure of the effective plastic strain by matching the computed minimum perforation speed with the experimental one for flat PC plates of different thicknesses (see Sec. 4). Subsequently, this failure strain is used to characterize the effect of curvature on the resistance to perforation of panels with positive and negative curvatures and the edge length to the radius of curvature ratio varying between 0 and 2 . The effect of curvature on the penetration/perforation resistance of curved thermoelastoviscoplastic panels undergoing large plastic deformations prior to failure has not been studied before. This work differs from our earlier work [39] in which the panels were not perforated.

The rest of the paper is organized as follows: The initialboundary-value problem studied is described in Sec. 2, and the corresponding computational model is detailed in Sec. 3. Results for the low and the high velocity impacts of flat and curved PC panels and their comparisons with the test data available in the literature are presented and discussed in Secs. 3.2 and 4, respectively. The effect of curvature on the impact response of panels of various thicknesses is investigated in Sec. 5. Conclusions from this work are summarized in Sec. 6.

\section{Mathematical Model}

Figure 1 depicts a schematic sketch of the plate-impactor system before the impactor contacts the panel, the $(\mathrm{O} x z)$ rectangular Cartesian coordinate system used to describe panel's deformations, and a positively and a negatively curved panel. The clamped panel has thickness $h$, and its curved midsurface has sides of lengths $L_{1}$ and $L_{2}$. The panel center is impacted at normal incidence by a hemispherical-nosed steel circular cylinder of diameter d.

In the Lagrangian description of motion, transient deformations of the panel are governed by the following conservation laws:

$$
\begin{aligned}
& \text { mass : } \quad \rho J=\rho_{0} \\
& \text { linear momentum } \quad \rho_{0} \dot{\mathbf{v}}=\hat{\nabla} \cdot \mathbf{T} \\
& \text { moment of momentum : } \quad \mathbf{T} \cdot \mathbf{F}^{\mathrm{T}}=\mathbf{F} \cdot \mathbf{T}^{\mathrm{T}}
\end{aligned}
$$

Here, $\rho$ and $\rho_{0}$ are the mass densities in the current and the reference configurations, respectively, $J=\operatorname{det}(\mathbf{F}), \quad \mathbf{F}=\partial \mathbf{x} / \partial \mathbf{X}$ is the deformation gradient mapping an infinitesimal line element emanating from a material point $\mathbf{X}$ in the reference position to a line element passing through its current location $\mathbf{x}$, a superimposed dot indicates the material time derivative, $\mathbf{v}$ is the velocity of a material point, $\mathbf{T}$ is the first Piola-Kirchhoff stress tensor related to the Cauchy stress tensor $\boldsymbol{\sigma}$ by $\mathbf{T}=J \boldsymbol{\sigma} \cdot \mathbf{F}^{-\mathrm{T}}$, and $(\widehat{\nabla})$ is the divergence operator with respect to $\mathbf{X}$.

For a few simulations at impact speeds used in Gunnarsson et al. [31] with the steel modeled as a Johnson-Cook material, we found that less than $1 \%$ maximum effective strain was induced in the impactor while deformations and/or failure of the PC panel were essentially unchanged. Therefore, in subsequent simulations for which results are reported below, the impactor was modeled as rigid that greatly reduced the computational cost. Khalili et al. [40] also showed that assuming the impactor to be rigid reduces the computation time with negligible effects on numerical results. The smooth impactor is assumed to have only translational motion governed by

$$
\dot{\mathbf{p}}=\mathbf{f}
$$

where $\mathbf{p}$ equals the linear momentum, and $\mathbf{f}$ the resultant force acting on the impactor. 
At points on clamped edges, the three displacement components are set equal to zero. At a free surface, the surface tractions vanish. On the smooth contact surface between the impactor and the panel, the following three continuity conditions are applied:

$$
\begin{array}{ll}
\text { normal velocity : } & {[[\dot{\mathbf{u}}]] \cdot \mathbf{n}=0} \\
\text { normal traction : } & {[[\mathbf{t}]] \cdot \mathbf{n}=0} \\
\text { tangential traction } & \mathbf{t} \times \mathbf{n}=0
\end{array}
$$

Here, $[[\mathbf{t}]]$ indicates the jump in the traction vector $\mathbf{t}$ across the contact surface, $\mathbf{n}$ is a unit normal to the contact surface, $\mathbf{u}$ is the displacement field, $\dot{\mathbf{u}}$ is the velocity field, and the symbol $\times$ denotes the cross product between two three-dimensional vectors. These conditions imply that there is no interpenetration between the impactor and the PC panel. However, the penalty method used in the numerical scheme to satisfy Eq. (3) allows tiny interpenetrations. At a point on the contact surface, the normal component of the velocity and the surface traction is continuous, and the tangential tractions vanish.

At time $t=0$, the panel is at rest, stress free, at the uniform temperature of $300 \mathrm{~K}$, and the moving impactor just contacts the top surface of the panel.

The thermoelastoviscoplastic response of the PC is modeled by the Mulliken and Boyce [5] constitutive relation with two modifications suggested by Varghese and Batra [18]. It is described in Ref. [39] where values of material parameters are also listed.

\section{Computational Model}

3.1 Implementation. We use the commercial FE software LSDYNA in which the constitutive relation for the PC has been implemented as a user-defined subroutine written in FORTRAN. The verification of the implementation has been described by Varghese and Batra [18], who showed that for simple tensile deformations, the computed true axial stress versus true axial strain agreed well with the corresponding experimental results till the axial strain of 0.8 . It has been assumed here that the same values of material parameters can be used till the effective plastic strain of 3.0.

An "unstructured" FE mesh used to discretize the panel gives better convergence rate versus the computational cost than a "structured" mesh [40]. We use hexahedral elements with onepoint integration rule for evaluating element matrices, and control zero-energy deformation (or "hourglass") modes with the Belytschko-Bindeman algorithm. For all the simulations, the energy of the hourglass modes is found to be less than $5 \%$ of the total internal energy that equals the sum of the elastic energy, the kinetic energy (KE), and the plastic dissipation. The accuracy of LS-DYNA's contact algorithm was checked in Ref. [39] for a quasistatic Hertz contact problem.

As described for a flat plate in the Appendix of Ref. [39], the FE mesh pattern in the $x y$-plane is obtained by partitioning the plate along its diagonals. Then, each quarter of the plate is partitioned by a $12.7-\mathrm{mm}$ radius circle centered at the impact point. The circle circumference and each edge of the quarter plate are discretized with 38 uniform elements. A plate diagonal with points located more than $12.7 \mathrm{~mm}$ from the plate center is divided into 48 segments of different lengths with the ratio of the length of the smallest segment located near the circle to that of the largest segment located at the corner equal to 20. Each layer has 11,628 elements, and 11, 15, 18, 29, and 39 layers are used for the $3.0,4.45,5.85,9.27$, and $12.32 \mathrm{~mm}$ thick plates, respectively. A similar mesh generation strategy is adopted for curved panels.

For each impact problem studied, results were computed with at least two FE meshes. The FE mesh A was uniformly refined to obtain FE mesh B having at least $30 \%$ more nodes than those in mesh A. The process was repeated till the maximum reaction force, and the energy dissipation computed with two successive meshes differed by less than $10 \%$. Convergence of these two quantities does not imply the convergence of either the penetration depth or of stresses at a point.

3.2 Validation of the Model. We simulated test configurations of Gunnarsson et al. [31,41,42], who used the digital image correlation technique to experimentally measure back-surface deflections of clamped $254 \mathrm{~mm}$ square flat PC plates of thickness varying between 3 and $12.32 \mathrm{~mm}$ impacted by a $104 \mathrm{~g}$ steel impactor of radius $6.35 \mathrm{~mm}$ at speeds ranging from 10 to $50 \mathrm{~m} / \mathrm{s}$. The total length of the impactor equals $12.7 \mathrm{~mm}$. In Ref. [42], the authors provide time histories of the deflection of the center of the rear face of $3,4.45,5.85,9.27$, and $12.32 \mathrm{~mm}$ thick panels, while in Ref. [41] they give the deformed profiles of the rear face of the $5.60-\mathrm{mm}$ thick panel. The measured and the computed maximum deflections listed in Table 2 reveal that the largest difference, $10.3 \%$, between them is for the $12.32-\mathrm{mm}$ thick panel impacted at $40 \mathrm{~m} / \mathrm{s}$. The impact speeds given in Table 2 are approximate since a range of impact speeds was given for some of the tests.

As detailed in Ref. [39], the computational and experimental force-time histories and the deformed shapes are found to compare well with each other. The energy balance for a perforation problem described at the end of Sec. 4 also validates the model.

\section{Critical Strain for Ductile Failure}

We assume that a material point fails when the equivalent plastic strain, $\varepsilon_{i}^{\mathrm{p} 1}, i=\alpha, \beta$, in either phase $\alpha$ or phase $\beta$ (see Ref. [39] where all the symbols are defined) given by

$$
\begin{aligned}
\varepsilon_{i}^{\mathrm{p} 1} & =\int_{\tau=0}^{t} \sqrt{\frac{2}{3} \widetilde{\mathbf{D}}_{i}^{\mathrm{p}}: \widetilde{\mathbf{D}}_{i}^{\mathrm{p}}} d \tau=\int_{\tau=0}^{t} \sqrt{\frac{2}{3}} \dot{\gamma}_{i}^{\mathrm{p}} d \tau \approx \sum_{n} \sqrt{\frac{2}{3}} \dot{\gamma}_{i}^{\mathrm{p}} \Delta t, \quad i=\alpha, \beta \\
\varepsilon^{\mathrm{p} 1} & =\max \left(\varepsilon_{\alpha}^{\mathrm{p} 1}, \varepsilon_{\beta}^{\mathrm{p} 1}\right)
\end{aligned}
$$

equals 3; this is discussed below. As implied by the last expression on the right-hand side of Eq. (4), the integral is evaluated by assuming that $\dot{\gamma}_{i}^{\mathrm{p}}$ is constant during the time increment $\Delta t$.

Gunnarsson et al. [42] experimentally found the $V_{50}$ of 3.00 , 4.45 , and $5.85 \mathrm{~mm}$ thick plates to be, respectively, 62, 72, and $80 \mathrm{~m} / \mathrm{s}$ with an accuracy of about $1 \mathrm{~m} / \mathrm{s}$. However, they did not

Table 2 Comparison of the experimental [42] and the computed maximum deflections of the centroid of the back surface of clamped PC plates

Approximate impact velocity $(\mathrm{m} / \mathrm{s})$

10

Experimental (computed) maximum deflection in $\mathrm{mm}$, and \% difference between the two values

Panel thickness (mm)

$$
\begin{array}{cl}
13.2(13.0)[-1.5] & 16.1(17.1)[6.2] \\
9.4(9.0)[-4.3] & 12.9(13.1)[1.6] \\
6.5(7.1)[9.2] & 10.9(10.2)[-6.4]
\end{array}
$$

5.85

9.27

12.32

$\begin{array}{lcl}3.00 & 13.2(13.0)[-1.5] & 16.1(17.1)[6.2] \\ 4.45 & 9.4(9.0)[-4.3] & 12.9(13.1)[1.6] \\ 5.85 & 6.5(7.1)[9.2] & 10.9(10.2)[-6.4] \\ 9.27 & & \end{array}$

$19.2(19.0)[-1.0]$
$11.3(12.1)[7.1]$ $8.7(9.6)[10.3]$
$22.0(22.7)[3.2]$

$14.0(14.8)[5.7]$

$10.7(11.3)[5.6]$ 
Table 3 Simulation results for $3,4.45$, and $5.85 \mathrm{~mm}$ thick plates at various impact speeds ( $\mathrm{Y}=$ perforation)

\begin{tabular}{|c|c|c|c|c|c|c|}
\hline \multirow[b]{2}{*}{$\varepsilon^{\mathrm{pl}, \mathrm{f}}$} & \multirow[b]{2}{*}{$h(\mathrm{~mm})$} & \multicolumn{5}{|c|}{ Ratio of impact speed to experimental $V_{50}$} \\
\hline & & 0.90 & 0.95 & 1.00 & 1.05 & 1.10 \\
\hline \multirow[t]{3}{*}{2.5} & 3.00 & $\mathrm{~N}$ & $\mathrm{Y}$ & $\mathrm{Y}$ & $\mathrm{Y}$ & $\mathrm{Y}$ \\
\hline & 4.45 & $\mathrm{~N}$ & $\mathrm{Y}$ & $\mathrm{Y}$ & $\mathrm{Y}$ & $\mathrm{Y}$ \\
\hline & 5.85 & $\mathrm{~N}$ & $\mathrm{Y}$ & $\mathrm{Y}$ & $\mathrm{Y}$ & $\mathrm{Y}$ \\
\hline \multirow[t]{3}{*}{2.75} & 3.00 & $\mathrm{~N}$ & Y & $\mathrm{Y}$ & Y & $\mathrm{Y}$ \\
\hline & 4.45 & $\mathrm{~N}$ & Y & $\mathrm{Y}$ & $\mathrm{Y}$ & $\mathrm{Y}$ \\
\hline & 5.85 & $\mathrm{~N}$ & $\mathrm{~N}$ & $\mathrm{Y}$ & $\mathrm{Y}$ & $\mathrm{Y}$ \\
\hline \multirow[t]{3}{*}{3.0} & 3.00 & $\mathrm{~N}$ & $\mathrm{~N}$ & $\mathrm{Y}$ & $\mathrm{Y}$ & $\mathrm{Y}$ \\
\hline & 4.45 & $\mathrm{~N}$ & Y & $\mathrm{Y}$ & $\mathrm{Y}$ & $\mathrm{Y}$ \\
\hline & 5.85 & $\mathrm{~N}$ & $\mathrm{~N}$ & $\mathrm{Y}$ & $\mathrm{Y}$ & $\mathrm{Y}$ \\
\hline \multirow[t]{3}{*}{3.25} & 3.00 & $\mathrm{~N}$ & $\mathrm{~N}$ & $\mathrm{~N}$ & $\mathrm{~N}$ & $\mathrm{~N}$ \\
\hline & 4.45 & $\mathrm{~N}$ & $\mathrm{~N}$ & $\mathrm{~N}$ & $\mathrm{Y}$ & $\mathrm{Y}$ \\
\hline & 5.85 & $\mathrm{~N}$ & $\mathrm{~N}$ & $\mathrm{~N}$ & $\mathrm{Y}$ & $\mathrm{Y}$ \\
\hline \multirow[t]{3}{*}{3.5} & 3.00 & $\mathrm{~N}$ & $\mathrm{~N}$ & $\mathrm{~N}$ & $\mathrm{~N}$ & $\mathrm{~N}$ \\
\hline & 4.45 & $\mathrm{~N}$ & $\mathrm{~N}$ & $\mathrm{~N}$ & $\mathrm{~N}$ & $\mathrm{Y}$ \\
\hline & 5.85 & $\mathrm{~N}$ & $\mathrm{~N}$ & $\mathrm{~N}$ & $\mathrm{Y}$ & $Y$ \\
\hline
\end{tabular}

mention repeatability of their tests. The $V_{50}$ equals the impact speed such that for randomly selected impact speeds around it, complete perforation occurs for $50 \%$ of the impacts. In the present deterministic study, unless one introduces randomness in material properties and plate geometries, there is one minimum impact speed for full perforation. Since Gunnarsson et al. [42] did not report all the speeds used to find the $V_{50}$, we set the experimental $V_{50}$ equal to the impact speed for which the target is just perforated. These results are used to find the effective plastic strain at failure, $\varepsilon^{\mathrm{p} 1 \mathrm{f}}$. For these three plates, taking $\varepsilon^{\mathrm{p} 1, \mathrm{f}}=2$ gave numerically predicted perforation speeds that were nearly $75 \%$ of their test values, i.e., considerably less than their experimental values. For different values of $\varepsilon^{\mathrm{p} 1, \mathrm{f}}$ and impact speeds varying between $90 \%$ and $110 \%$ of the experimental minimum perforation speed, we have listed in Table 3 whether or not plates were perforated in the simulations. We note that $\varepsilon^{\mathrm{p} 1, \mathrm{f}}=3$ provides a satisfactory agreement between the numerical and the experimental results since the perforation velocity of the plates can be predicted with less than $10 \%$ error for the $4.45-\mathrm{mm}$ thick plate and with less than $5 \%$ error for the other two thicknesses considered. Thus, $\varepsilon^{\mathrm{p} 1, \mathrm{f}}=3$ is used in the rest of the paper. Failed elements are deleted from the analysis domain thereby creating a void. The coalescence of these failed elements results in either a hole or a crater.

Time histories of the impactor KE and of the internal, the kinetic, and the eroded energies for the 5.85-mm thick PC plate impacted at $100 \mathrm{~m} / \mathrm{s}$ are exhibited in Fig. 2. It is interesting to note that for this problem, the erosion energy begins to increase (i.e.,

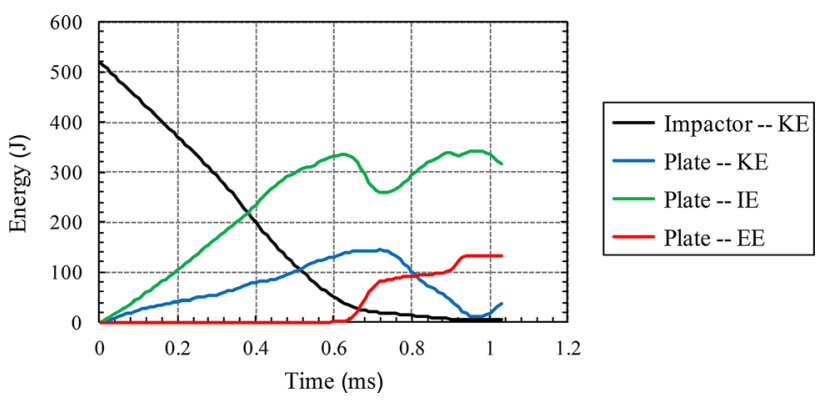

Fig. 2 Time histories of the impactor KE and of the plate internal, kinetic, and erosion energies for the impact of the $5.85-\mathrm{mm}$ thick flat PC plate at $100 \mathrm{~m} / \mathrm{s}$. KE, kinetic energy; IE, internal (elastic + plastic) energy; and EE, eroded energy. elements start failing and hence getting deleted) when the impactor KE has decreased to a small value. The decrease in the plate internal energy concurrent with an increase in the erosion energy suggests that some of the plate strain energy is used to erode additional elements and increase the plate KE. Subsequently, the plate $\mathrm{KE}$ is converted into the plate internal energy and the erosion energy till the plate motion considerably slows down and the plate has been perforated. The sum of the three energies of the plate nearly equals the initial KE, $520 \mathrm{~J}$, of the smooth rigid impactor.

Factors affecting the impact speed needed to just perforate a plate include the rigid impactor nose shape, penetrator length, target material properties, target areal density, boundary conditions, friction at the penetrator/target interface, and the penetrator nose radius relative to plate dimensions. For problems studied here, the hemispherical nose of the penetrator and the penetrator length is fixed, the target/penetrator interface is smooth, the target plate edges are clamped, the length of each side of the midsurface of the square target panel equals $254 \mathrm{~mm}$, and the penetrator radius equals $6.35 \mathrm{~mm}$. Thus, plate length/penetrator diameter equals $\sim 21$. The maximum elastic wave speed in the PC equals $\sim 1.18$ $\mathrm{mm} / \mu \mathrm{s}$. Thus, the elastic wave travels from the impact point to the plate edge in $\sim 107 \mu \mathrm{s}$. Since failure times for thin panels studied are $\sim 1 \mathrm{~ms}$, boundary conditions at the edges can affect deformations near the impact point. For problems studied here, the panel thickness/penetrator length varies from 0.24 to 1 . Thus, results for penetration into thick targets are not applicable here. Another nondimensional parameter often used to characterize the effect of inertia forces for penetration problems is: target mass density $\times$ impact speed $^{2} /$ target yield strength. For the PC and the impact speed of $100 \mathrm{~m} / \mathrm{s}$, it equals $\sim 0.1$. Thus, one could potentially ignore effects of inertia forces on panel's deformations. However, the present analysis includes effects of inertia forces.

Upon impact at $V \mathrm{~m} / \mathrm{s}$, the target point directly underneath the penetrator nose-tip has the maximum pressure = acoustic impedance of the $\mathrm{PC} \times$ impact speed $=1419 \mathrm{~V} \mathrm{kPa}$ and undergoes large deformations. The computed maximum pressure soon after impact does satisfy this relation and provides credence to the results reported here. As the impactor indents into the target, the reaction force is distributed on the penetrator nose that retards its motion. The impactor KE versus time depicted in Fig. 2 reveals that it varies nearly linearly from $520 \mathrm{~J}$ at $t=0$ to $100 \mathrm{~J}$ at $t=0.5 \mathrm{~ms}$. Thus, $\frac{1}{2} m v^{2}=520-840,000 t$, where time $t$ is in seconds, $m=0.104 \mathrm{~kg}$ is the penetrator mass, the penetrator deceleration equals $8076 / V \mathrm{~mm} / \mathrm{s}^{2}$, and the instantaneous force at $t=0$ resisting impactor's motion equals $8.4 \mathrm{kN}$. This data should help experimentalists appropriately design sensors to be used in tests.

We recall that for infinitesimal deformations for which the material response can be considered as linear elastic, the panel bending stiffness is proportional to $h^{3}(h=$ panel thickness). For a rigid perfectly plastic material, the bending moment at a fully plastically deformed cross section (plastic hinge) is proportional to $h^{2}$. The force required to uniformly stretch a cross section is proportional to $h$. For finite deformations, the hoop strain has terms involving $1 / R^{2}$. The consideration of material nonlinearities complicates the quantification of different modes of deformation in the present transient problem.

For relatively high-speed impacts on thermoviscoplastic targets, the material strength and the equation of state influence target's deformations in the contact region between the smooth impactor and the target, e.g., see Ref. [43]. For steady-state penetration of a rigid hemispherical-nosed cylindrical penetrator into a thermoviscoplastic target, it is shown in Ref. [44] that the friction force has minimal effect on the normal stress but significantly affects the tangential velocity of target particles flowing over the penetrator nose. For the present impact problem, plots of the time history of the total contact (or the reaction) force acting on the impactor depicted in Fig. 3 for three values of the coefficient of friction reveal that the Coulomb friction at the penetrator/target contact surface has negligible effect on the impactor retardation. The consideration of frictional effects did not materially affect the distribution of the effective plastic strain 


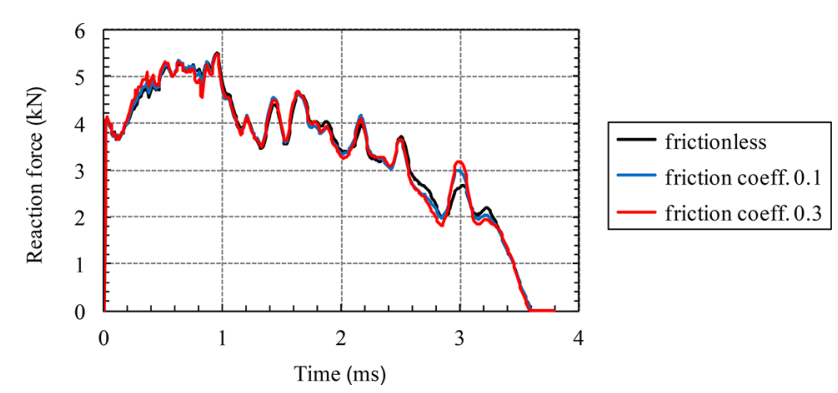

Fig. 3 Contact force as a function of time for 4.45-mm thick positively curved PC panels with $R=127 \mathrm{~mm}$ impacted at $72 \mathrm{~m} /$ $s$ with different values of the friction coefficient

in the region adjoining the penetrator/target interface. As shown in Fig. 4, the impactor did not penetrate into the panel.

Sensitivity studies for impact problems similar to those being studied here but involving no perforation reported in Ref. [45] have revealed that five parameters with the highest mean effects on the stress-strain curves for the PC deformed at 5000/s in uniaxial compression are Young's modulus, Poisson's ratio, the activation energies of the two phases in the constitutive relation for the $\mathrm{PC}$, and a stress softening parameter. The latter three parameters strongly influence the energy dissipated in the PC panel, and the first two affect the reaction force. For the present problems, we anticipate that all the five material parameters and the value of the failure strain noticeably influence the $V_{50}$ for the panels.

As should be clear from the results listed in Table 3, the strain at failure plays a critical role in determining the minimum impact speed required to perforate the target. In the work reported below, only an approximate value of the minimum speed required to perforate the target has been computed since the emphasis is on delineating effects of panel curvature/panel thickness ratio on the impact performance when the impact speed is close to the minimum perforation speed. The experimental $V_{50}$ values are also approximate with unknown certainties.

Once the maximum effective plastic strain in an element reaches the critical value of 3 , it is deleted and stresses are redistributed in the region surrounding the deleted element. The instant of element deletion depends upon the impact speed and the target thickness to curvature ratio with other factors kept fixed. Upon penetration of the impactor into the target, the target thickness under the penetrator nose varies with time that also affects deformation mechanisms of the plate. Thus, the dominant deformation mechanisms vary with time as the impactor pushes its way through the target.

\section{Perforation of Panels}

For impact speed $=72 \mathrm{~m} / \mathrm{s}$, we have plotted in Fig. 4 deformed shapes and the plastic strain distribution in the flat and the curved panels with $h=4.45 \mathrm{~mm}$ and $R= \pm 127 \mathrm{~mm}$. Both the flat and the

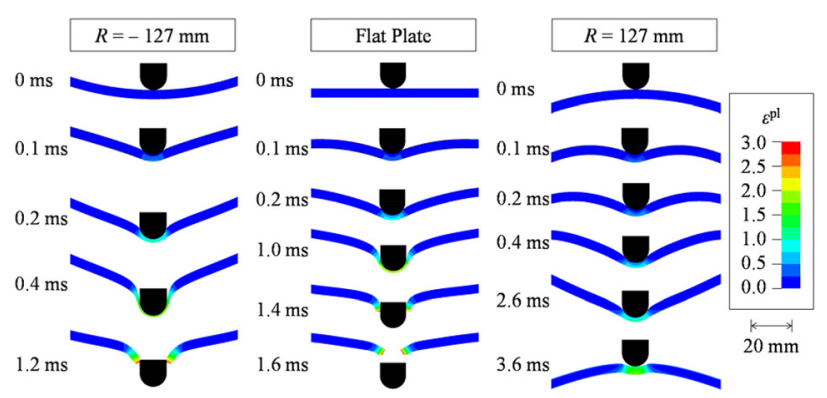

Fig. 4 Deformed shapes and plastic strain distributions in 4.45-mm thick panels for $72 \mathrm{~m} / \mathrm{s}$ impact speed

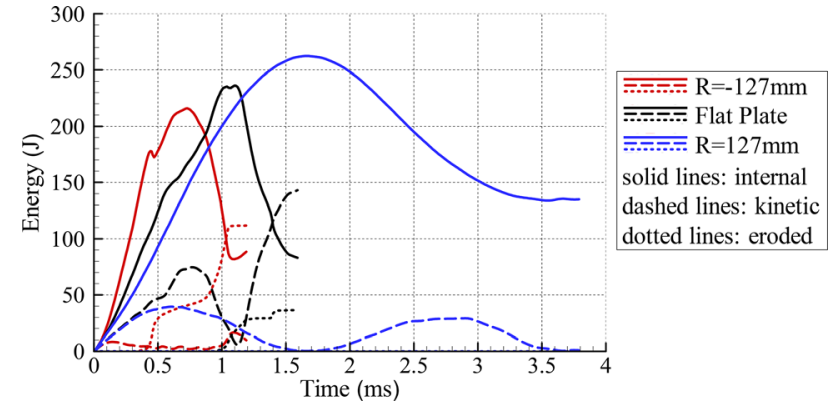

Fig. 5 Time histories of the internal, the kinetic, and the eroded energies of the $4.45-\mathrm{mm}$ thick panels for $72 \mathrm{~m} / \mathrm{s}$ impact velocity

panel with the negative curvature are perforated but that with the positive curvature is not perforated. The deformed shapes of the panels at different instants of time after impact show that the positively curved panel with $R=127 \mathrm{~mm}$ undergoes large deflections before the impactor bounces back, while the material of the panel with $R=-127 \mathrm{~mm}$ undergoes significant plastic deformations without the panel bouncing back. The material underneath the impactor nose is significantly (negligibly) stretched and wrapped around the impactor for the panel with $R=-127 \mathrm{~mm}(127 \mathrm{~mm})$. The panel with the negative curvature is perforated at $\sim 1.2 \mathrm{~ms}$ after impact, and the flat plate at $\sim 1.6 \mathrm{~ms}$. Thus, the panel with the positive curvature provides more impact resistance than the other two panels. The problem of finding the curvature/thickness ratio for maximizing the perforation resistance for a given impactor, impactor nose shape, and its speed is left for a future study. Note that the length of each side of the midsurface of the square target panel equals $254 \mathrm{~mm}$, thus side length/panel curvature is proportional to $R$.

Time histories of the internal, the kinetic, and the eroded energies of the plates are shown in Fig. 5. The internal energy equals the sum of energies of the elastic and the plastic deformations, while the eroded energy equals the internal and the KE of the solid elements that failed and were subsequently removed from the analysis. These results suggest that the KE of the plate with the negative curvature remains negligibly small as compared to those of the flat and the positive curvature panels. The maximum eroded energy is significantly more for the negatively curved panel than that for the other two panels and is essentially zero for the positively curved panel since no element failed for it. Elements in the negatively curved panel start eroding at $t=\sim 0.4 \mathrm{~ms}$ but in the flat panel at $\sim 1 \mathrm{~ms}$. The larger value of the total eroded energy suggests that a larger volume of the material is eroded in the negatively curved panel than that in the flat panel. The maximum internal energy is highest for the positively curved panel and least for the negatively curved panel. Factors contributing to the total internal energy are the intensity of the elastic and plastic deformations and how much of the volume is plastically deformed. The results exhibited in Fig. 4 suggest that the maximum plastic strain induced in the panel of positive curvature is less than that induced in the other two panels. Also, the volume of the plastically deformed material for the positive curvature panel is the least

Table 4 Maximum temperature rise in the panels excluding that in the eroded material

\begin{tabular}{lccccccc}
\hline \hline$R(\mathrm{~mm})$ & -127 & -254 & -508 & Flat & 508 & 254 & 127 \\
\hline$h=3.00 \mathrm{~mm}, v=62.5 \mathrm{~m} / \mathrm{s}$ & 70 & 66 & 60 & 63 & 64 & 52 & 37 \\
$h=4.45 \mathrm{~mm}, v=72 \mathrm{~m} / \mathrm{s}$ & 63 & 69 & 66 & 64 & 65 & 50 & 40 \\
$h=5.85 \mathrm{~mm}, v=80 \mathrm{~m} / \mathrm{s}$ & 69 & 82 & 92 & 90 & 68 & 42 & 56 \\
$h=9.27 \mathrm{~mm}, v=100 \mathrm{~m} / \mathrm{s}$ & 72 & 78 & 75 & 76 & 76 & 57 & 80 \\
$h=12.32 \mathrm{~mm}, v=115 \mathrm{~m} / \mathrm{s}$ & 78 & 81 & 112 & 91 & 92 & 89 & 86 \\
\hline \hline
\end{tabular}




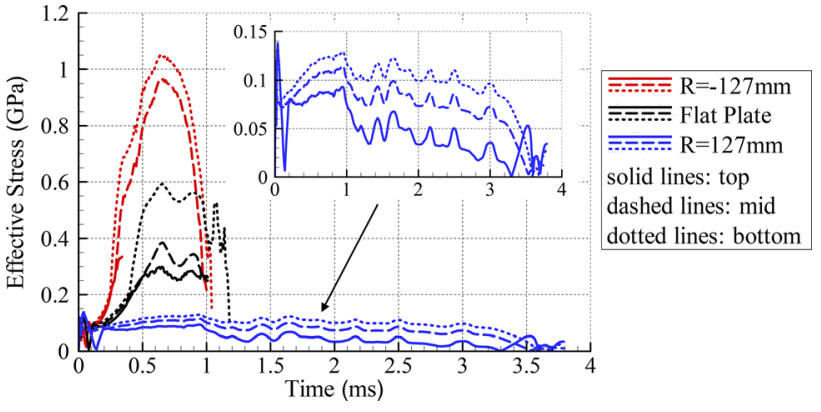

Fig. 6 Time histories of the effective stress at the centers of the plate top, the mid, and the bottom faces of the panels with $h=4.45 \mathrm{~mm}$ and different curvatures (impact speed $=72 \mathrm{~m} / \mathrm{s}$ )

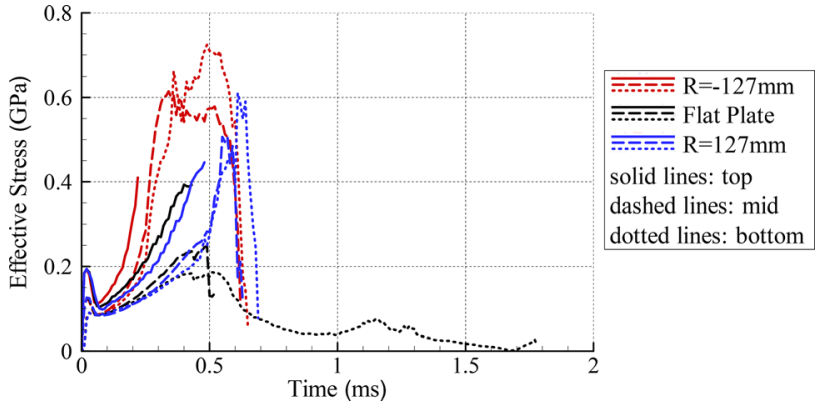

Fig. 7 Time histories of the effective stress at the center of the 12.32-mm thick plate's top, mid, and bottom faces and different curvatures for the impact speed of $115 \mathrm{~m} / \mathrm{s}$ among those for the three panels. These observations suggest that the strain energy of elastic deformations in the positively curved panel is the highest among that for the three panels. The maxima in the internal energies of the positive, the zero, and the negative curvature panels occur, respectively, at $t=1.7,1.1$, and $0.75 \mathrm{~ms}$ after impact, and their values equal 270, 240, and $220 \mathrm{~J}$, respectively.

5.1 Temperature Rise. For the 35 simulations involving panels of five different thicknesses and three curvatures, the maximum temperature rise at any point in the plate, excluding the eroded material, is listed in Table 4 , and is found to be about $112^{\circ} \mathrm{C}$. For the room temperature $25^{\circ} \mathrm{C}$, the maximum temperature reached in the panel is below the melting temperature $\left(\sim 155^{\circ} \mathrm{C}\right)$ of PC. However, it is important to use constitutive relations similar to the one employed here that incorporate the temperature dependence of the mechanical response of the material. The maximum temperature rise confirms that the peak effective plastic strain in the negatively curved panel is more than that in the flat and the corresponding positively curved panels.

5.2 Stresses Developed. In Fig. 6, we show time histories of the effective stress at the center of the top-, the mid-, and the bottom-surfaces of the plates of Fig.4. For $R=-127 \mathrm{~mm}$, the curve corresponding to the top surface ends at about $0.4 \mathrm{~ms}$, the time when the solid element at the center of the top surface fails. Subsequently, the failure simultaneously propagates outward and through the panel thickness. An element at the midsurface fails at $t=1 \mathrm{~ms}$ and that at the bottom face at $t=1.05 \mathrm{~ms}$. One reason for the very little time elapsed between failures at the mid- and the bottom-surfaces is that the material had already been considerably damaged by the time the element at the midsurface failed. Similar remarks and explanations apply to deformations for the flat plate for which an element on the top surface fails at $1 \mathrm{~ms}$ and that at the bottom surface at $1.2 \mathrm{~ms}$. It is also obvious that the maximum magnitude of the effective stress in the panel with $R=127 \mathrm{~mm}$ is considerably less than that for the other two panels which evinces the beneficial effect of the positive curvature on the impact response of the $4.45-\mathrm{mm}$ thick panel. The difference between the maximum effective stresses on the top and the bottom surfaces is the largest for the panel with $R=-127 \mathrm{~mm}$ and the smallest for the panel with $R=127 \mathrm{~mm}$.

For the $12.32-\mathrm{mm}$ thick panels impacted at $115 \mathrm{~m} / \mathrm{s}$, we exhibit in Fig. 7 the time histories of the effective stress at points located at the center of the plate top, the middle, and the bottom surfaces. On the top (middle and bottom) surface, the maximum effective stress for the positively (negatively) curved panel is the largest of the three. On the middle and the bottom surfaces, the maximum effective stress for the flat plate is noticeably less than that for the two-curved plates. For the three panels, the material failure initiates at the impact point on the top surface and propagates to the back surface of the plate. Although some material of the flat plate failed, it was not completely perforated since elements located near its back surface did not fail.

In order to ascertain dominant deformation modes in the impacted plates, we define the time-averaged axial stress $T_{\text {axial }}$ at a point and its through-the-thickness average $T_{\text {axial,avg }}$ by
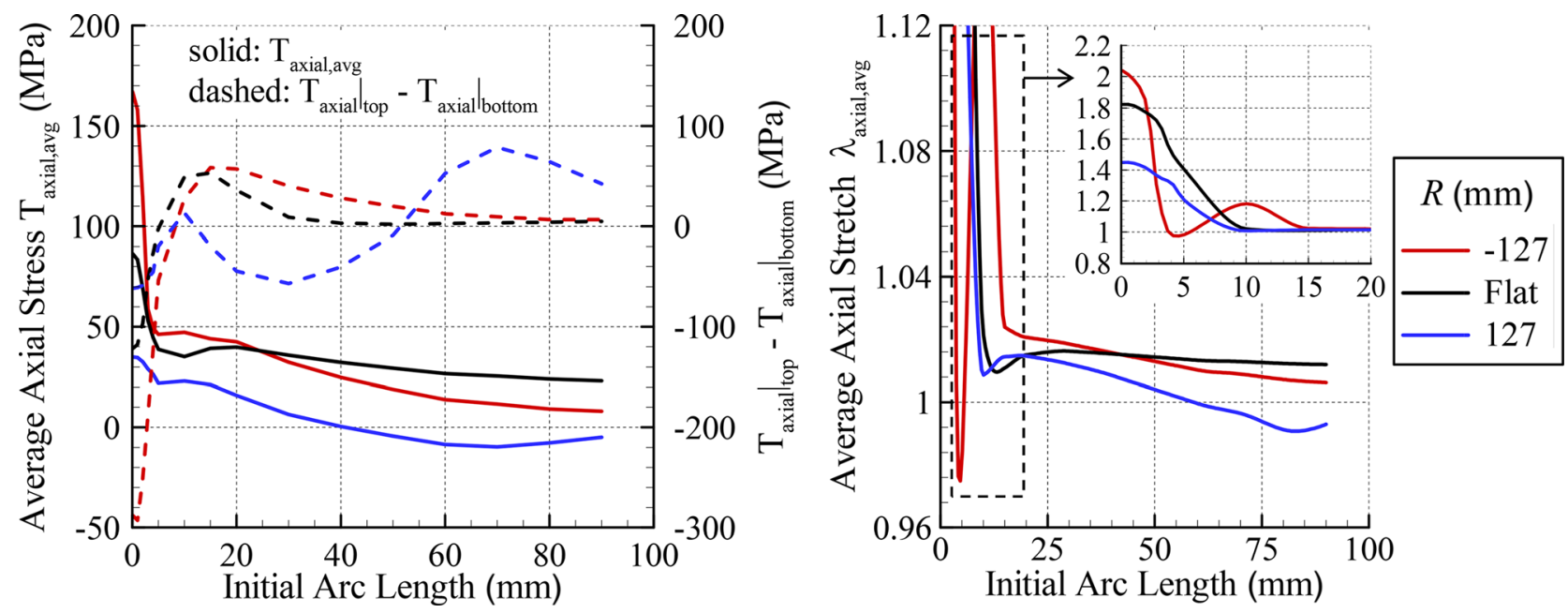

Fig. 8 Average axial stress and average axial stretch for the 4.45- $\mathrm{mm}$ thick panels and $72 \mathrm{~m} / \mathrm{s}$ impact velocity as a function of the initial arc length (measured from the panel center) 

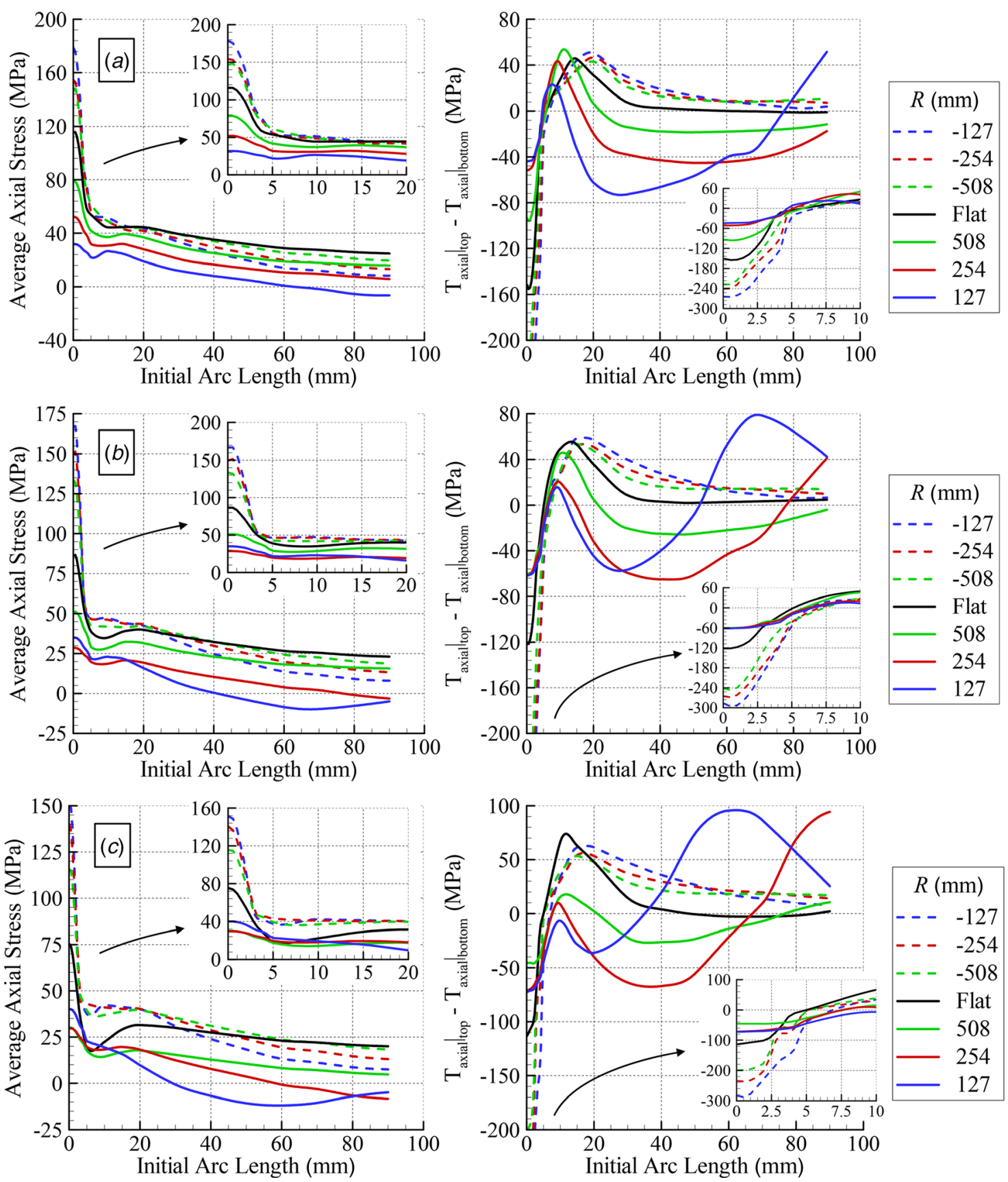

Fig. 9 Average axial stress and the difference between the axial stress on the top and on the bottom surfaces of the (a) $3 \mathrm{~mm},(b) 4.45 \mathrm{~mm},(c) 5.85 \mathrm{~mm},(d) 9.27 \mathrm{~mm}$, and (e) $12.32 \mathrm{~mm}$ thick panels as a function of the initial arc length measured from the panel centroid. For each panel thickness, the impact velocity equals the $V_{50}$ of the flat plate, i.e., $62.5,72,80,100,100$, and $115 \mathrm{~m} / \mathrm{s}$, respectively, for $(a)-(e)$.

$$
\begin{aligned}
\widehat{T}_{\text {axial }} & =\left[\left(\frac{\mathbf{F} \cdot \mathbf{N}}{\|\mathbf{F} \cdot \mathbf{N}\|}\right)^{\mathrm{T}} \cdot \mathbf{T} \cdot \mathbf{N}\right], \quad T_{\text {axial }}=\frac{1}{t_{f}} \int_{t=0}^{t_{f}} \widehat{T}_{\text {axial }} d t, \\
T_{\text {axial }, \text { avg }} & =\frac{1}{h} \int_{Z=-h}^{0} T_{\text {axial }} d Z
\end{aligned}
$$

Here, $T_{\text {axial }}$ is the axial stress at a material point averaged over time from the beginning of impact till the time $t_{f}$ when either the plate is fully perforated or the impactor finally separates from the plate, $\mathbf{T}$ is the first Piola-Kirchhoff stress tensor, and $\mathbf{N}$ is a unit vector normal to the local cross section in the undeformed configuration and pointing toward the panel center. The value of $t_{f}$ varies with the plate thickness and the impact speed, and the plate has not come to rest at time $t_{f}$.

For the flat and the two-curved panels, we have exhibited in Fig. 8 the spatial distribution of the average axial stress $T_{\text {axial,avg }}$ and of the average axial stretch $\lambda_{\text {axial,avg }}$ defined as

$$
\lambda_{\text {axial }}=\frac{1}{t_{f}} \int_{t=0}^{t_{f}} \lambda_{i_{0}} d t, \quad \lambda_{\text {axial,avg }}=\frac{1}{h} \int_{Z=-h}^{0} \lambda_{\text {axial }} d Z
$$

where $\lambda_{i_{0}}$ is the eigenvalue of the left Cauchy-Green tensor $\mathbf{B}$ corresponding to the eigenvector of $\mathbf{B}$ nearest to the vector $\mathbf{F} \cdot \mathbf{N}$. That is, 

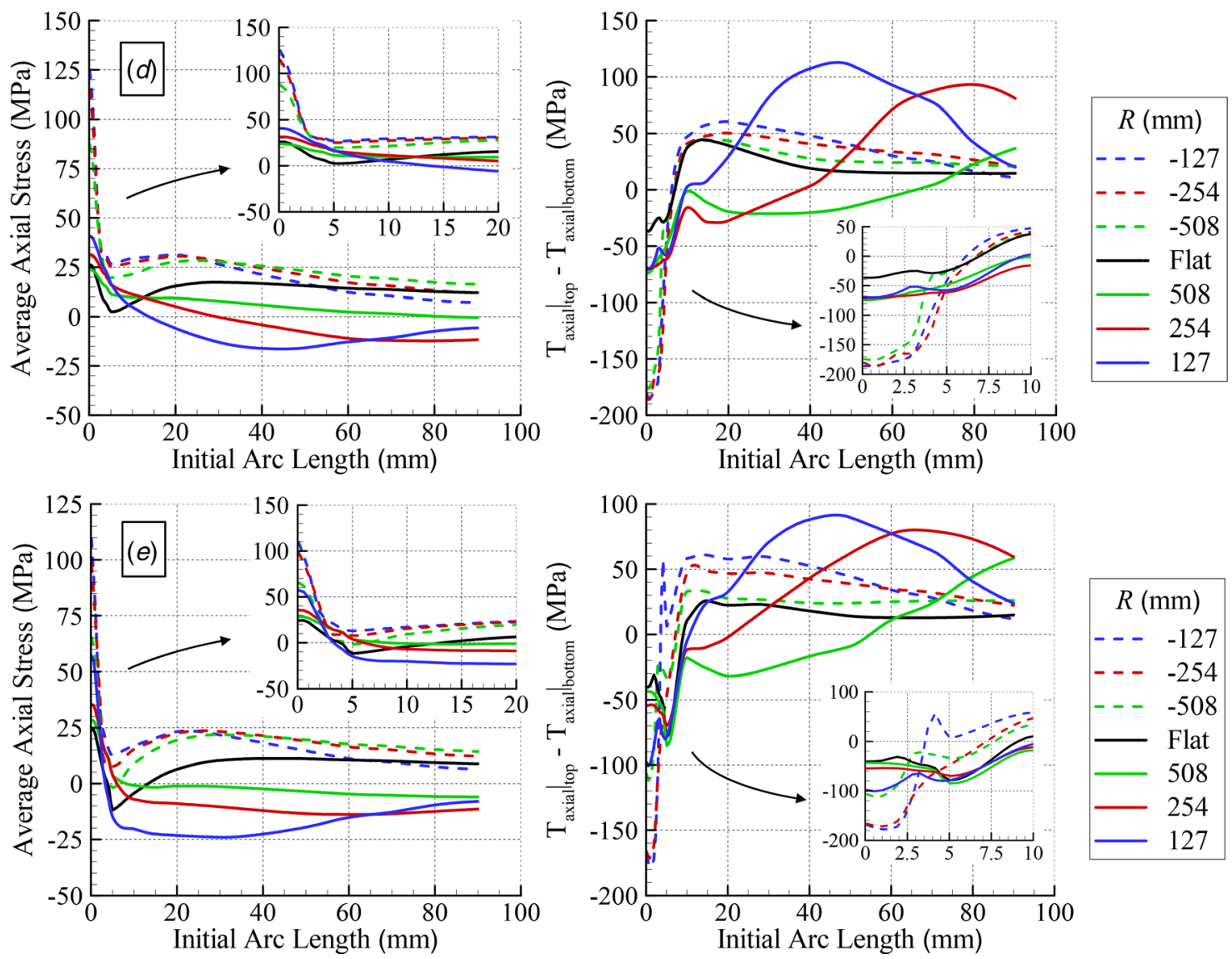

Fig. 9 (Continued)

$$
\begin{aligned}
i_{0} & =\underset{i}{\operatorname{argmax}}\left|\frac{\mathbf{F} \cdot \mathbf{N}}{\|\mathbf{F} \cdot \mathbf{N}\|} \cdot \mathbf{v}_{i}\right| \text { with } \mathbf{V}=\sqrt{\mathbf{B}}=\sqrt{\mathbf{F} \cdot \mathbf{F}^{\mathrm{T}}} \\
& =\sum_{i=1}^{3}\left(\lambda_{i} \mathbf{v}_{i} \otimes \mathbf{v}_{i}\right),\left\|\mathbf{v}_{i}\right\|=1
\end{aligned}
$$

This definition is motivated by the expectation that one eigenvector of $\mathbf{B}$ is tangent to the midplane of the deformed panel. For all the situations considered, it was found that the angle between $\mathbf{V}_{i_{0}}$ and $(\mathbf{F} \cdot \mathbf{N})$ was less than $25 \mathrm{deg}$ implying that those vectors were nearly collinear.

The results plotted in Fig. 8 (left) imply that the maximum average axial stress occurs at the panel centroidal axis, and the positive curvature decreases the average axial stress within the panel while the negative curvature increases it. The value of $T_{\text {axial,avg }}$ at the panel center for $R=127 \mathrm{~mm}$ is nearly one-half of that for the flat plate, and one-fourth of that of the panel with $R=-127 \mathrm{~mm}$. The value of the axial stretch $\lambda_{\text {axial,avg near the }}$ panel center of $R=127 \mathrm{~mm}$ is less than that for the panel with $R=-127 \mathrm{~mm}$. Therefore, the improvement in the impact resistance of a panel of positive curvature is due to the decrease in the average axial stress and the average axial stretch produced in it as compared to that in the flat plate. In other words, the central panel region under the impactor is stretched $\sim 50 \%$ (20\%) more for the negatively curved panel than that for the positively curved (flat) panel. We note that the difference between the axial stresses at points of intersection of a transverse normal with the top and the bottom surfaces of the panel near the centroidal axis is a very large negative number for the panel with $R=-127 \mathrm{~mm}$, while it is a positive small number for the two other panels.

Variations of the average axial stress $T_{\text {axial,avg }}$ with the curvilinear distance from the center of impact (measured in the undeformed configuration) are displayed in Figs. 9(a)-9(e) for plate

thicknesses between 3 and $12.32 \mathrm{~mm}$. We also give in the figure the difference between the values of $T_{\text {axial }}$ measured on the top and the bottom faces of the plate as a function of the distance from the plate centroidal axis. This provides some information about bending deformations of the plate since a positive value indicates that the plate is locally bent downward while a negative value indicates the opposite. In view of large plastic deformations induced, stresses are not proportional to the strains.

The PC panels are perforated due to the ductile failure of the material. The plastic deformations are highly localized as shown in Fig. 4, and the stress and the strain states in the vicinity of the impact point are critical for the impact resistance of the panels. The results depicted in Fig. 9 explain the smaller benefit of the positive curvature for the thicker panels since the decrease in the average axial stress near the impact point is less important. This explains, at least partially, the correlation between results depicted in Fig. 9 and those presented in Fig. 4. The value of the difference

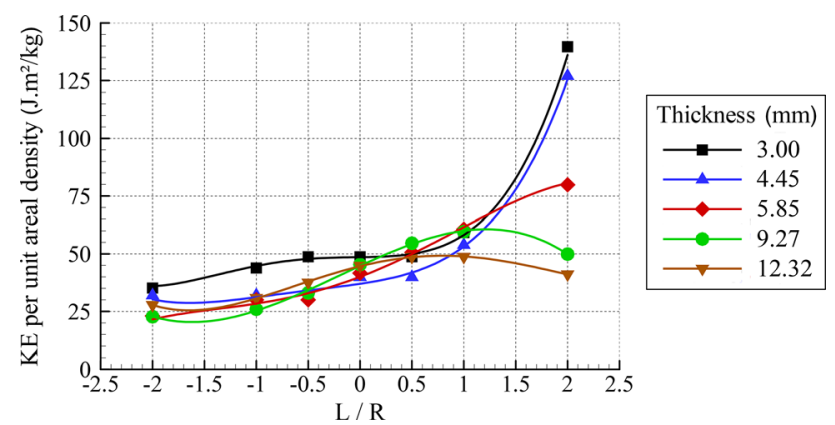

Fig. 10 Normalized impactor energy for perforation as a function of the panel curvature 

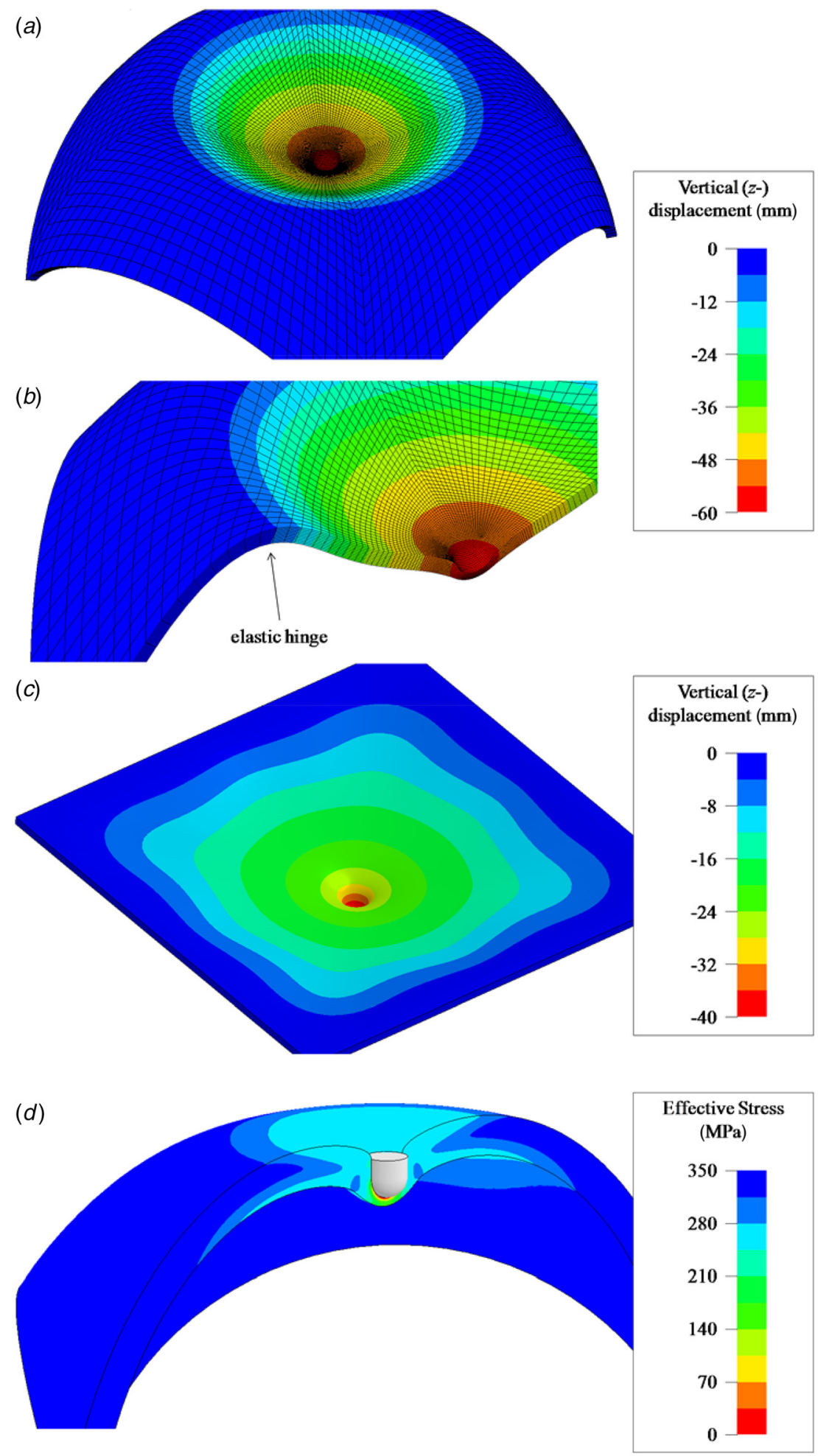

Fig. 11 For an impact speed of $72 \mathrm{~m} / \mathrm{s}$, the deformed shape in the 4.45-mm thick positively curved panel $(R=127 \mathrm{~mm})$ at $t=1.6 \mathrm{~ms}(a)$ and $(b)$, in the flat plate at $t=1 \mathrm{~ms}(c)$, and of positively curved $12.32-\mathrm{mm}$ thick panel at $t=0.4 \mathrm{~ms}(d)$. There is no hinge formed in both the flat plate and the positively curved thick panel.

between the axial stress on the top and the bottom faces does not seem to be correlated to the impact strength of the plates since even for the thicker panels the effect of positive curvature is considerable. Therefore, it seems that the profiles presented in Fig. 9 (right) do not explain the dependence of $V_{50}$ upon the panel curvature but rather indicate where and for which panels bending effects dominate over the in-plane stretching effects. The large differences between the axial stress on the top and the bottom faces near the impact point show that in the vicinity of the plate center (where there is contact with the impactor), the tensile inplane stress on the rear surface is considerably larger than that on the top surface. 


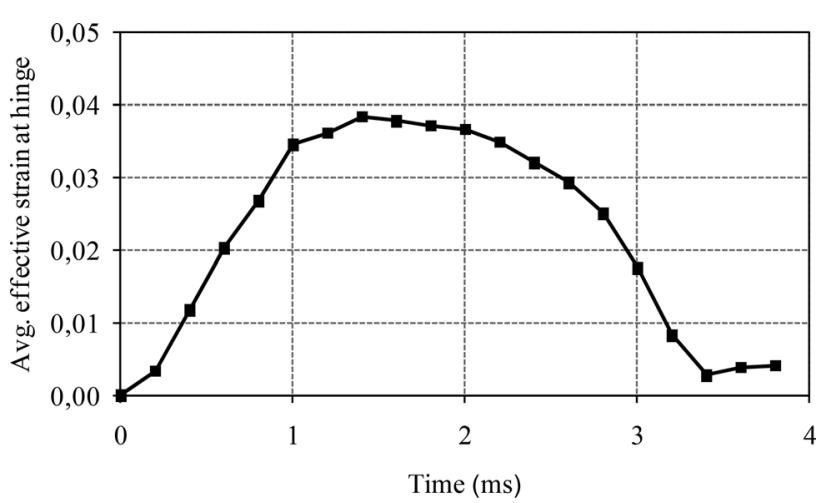

Fig. 12 Time history of the effective strain at the hinge in the $4.45-\mathrm{mm}$ thick positively curved panel $(R=127 \mathrm{~mm})$ impacted at $72 \mathrm{~m} / \mathrm{s}$

5.3 KE of Impactor Versus Panel's Curvature. The impactor KE normalized by the panel areal density (or the panel thickness since the volumetric mass density is constant) corresponding to the perforation velocity of the $3,4.45,5.85,9.27$, and $12.32 \mathrm{~mm}$ thick panels is plotted in Fig. 10 as a function of the panel midsurface length/radius of curvature. Recall that the midsurface length of all the panels equals $254 \mathrm{~mm}$. The filled squares, triangles, and circles correspond to the computed data points, while the solid curves are polynomial fits to these points. One can conclude from these plots that the perforation speed of a panel increases with an increase in its curvature. The curves for the 3and $4.45-\mathrm{mm}$ thick panels have different profiles from those for the 9.27- and $12.32-\mathrm{mm}$ thick panels, whereas that of the 5.85$\mathrm{mm}$ thick panel lies between those of the thinner and the thicker panels. For $L / R>1$, the impactor KE per unit areal density required to perforate the panel rapidly increases with an increase in $L / R$ for the 3- and 4.45-mm thick panels, but decreases for the thicker panels. For the five panels studied, the impactor KEs per unit areal density required to perforate them are clustered in a narrow range for $-2<L / R<1$.

5.4 Deformation Mechanisms. In an attempt to provide differences in deformation mechanisms of the $4.45-\mathrm{mm}$ thick positively curved and flat panels, we have plotted in Fig. 11 the deformed shape of the positively curved panel at $t=1.6 \mathrm{~ms}$ and of the flat plate at $t=1 \mathrm{~ms}$ for impact speed of $72 \mathrm{~m} / \mathrm{s}$. The plots also incorporate fringes of the effective strain on the top surface and through-the-thickness distributions on two cut sections. These results suggest that a considerable fraction of the central portion of the panel is bent by the impactor, and its $\mathrm{KE}$ is converted into the strain energy of the panel due to bending. Fringe plots of the effective strain on two cut sections exhibited in the figure reveal the formation of an elastic hinge around the impact point that divides the panel into two portions. Subsequent to the hinge formation, panel deformations are concentrated in the central part of the panel enclosed within the hinged surface. The time-history of the through-the-thickness average of the effective strain at the hinge is displayed in Fig. 12. It suggests that the maximum effective strain in the hinge is about $4 \%$ that reduces to nearly zero at $t=3.5 \mathrm{~ms}$ signifying that deformations at the hinge are elastic. No hinge is formed in the impacted flat plate. As compared to deflections of the positively curved panel, deflections of the flat plate are localized in the region surrounding the impact point and they
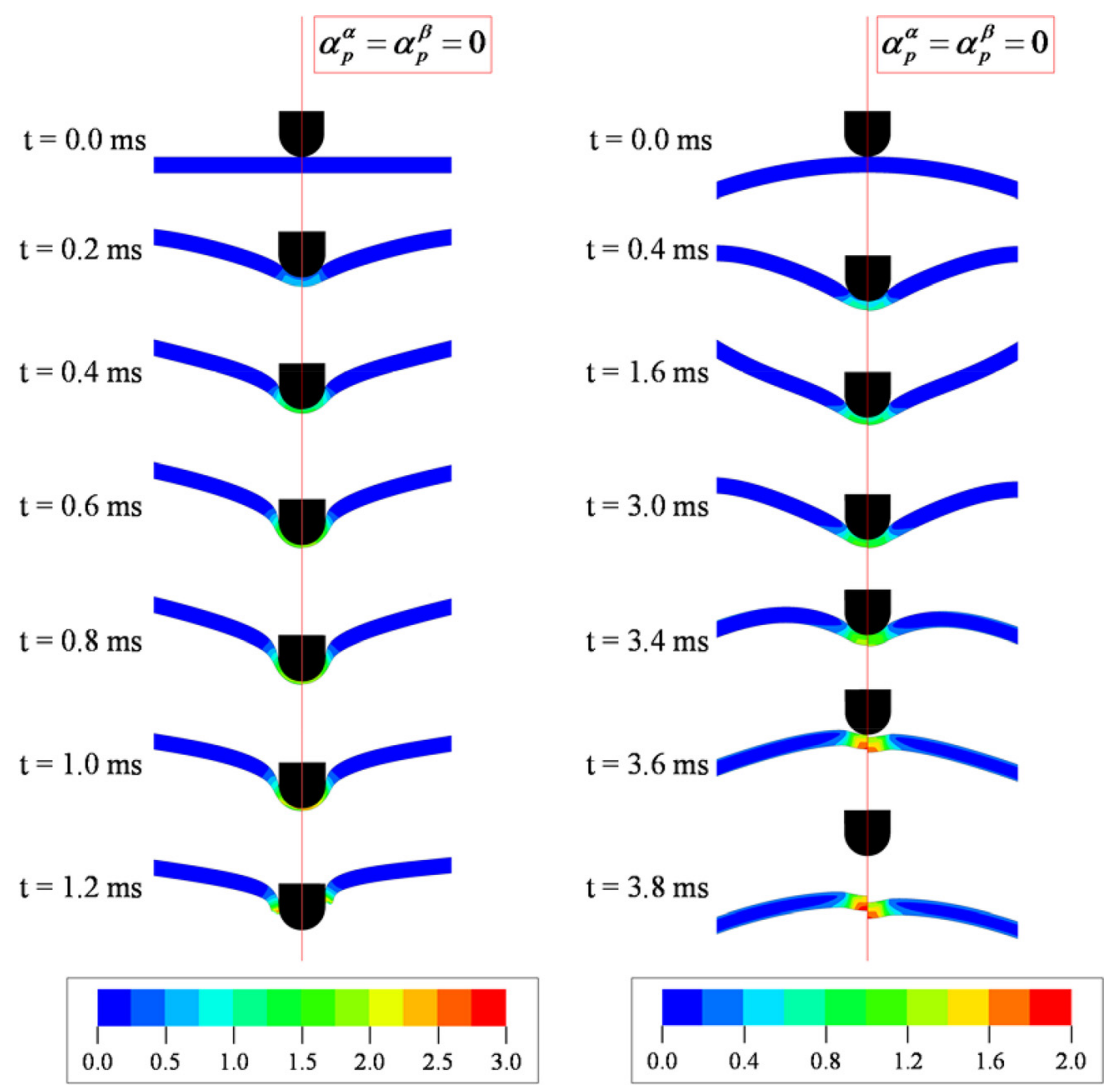

Fig. 13 Deformed shapes and plastic strain distributions in the 4.45-mm thick flat and positively curve panels $(R=127 \mathrm{~mm})$ for $72 \mathrm{~m} / \mathrm{s}$ impact speed with and without considering the effect of the pressure on the plastic multiplier in the PC constitutive relation. For each plot, the left part is obtained with pressure coefficients $\alpha_{p}^{\alpha}=0.128$ and $\alpha_{p}^{\beta}=0.254$, and the right part with $\alpha_{p}^{\alpha}=\alpha_{p}^{\beta}=0$. 
gradually decrease until the clamped boundaries of the plate. Thus, very little KE of the impactor is converted into the strain energy of the plate and most of it is used to plastically deform a small central portion of the plate that eventually fails.

We note that no elastic hinge formed in the 5.85, 9.27, and $12.32 \mathrm{~mm}$ thick panels irrespective of their curvatures.

5.5 Dependence Upon the Hydrostatic Pressure of Plastic Multipliers in the Constitutive Relation. The parameters $\alpha_{p}^{\alpha}$ and $\alpha_{p}^{\beta}$, respectively, for phases $\alpha$ and $\beta$ in the constitutive relation for the PC multiply the hydrostatic pressure and thus give the dependence of the effective plastic strain rate upon the effective deviatoric stress and the hydrostatic pressure. For metals, the effective plastic strain rate does not depend upon the hydrostatic pressure. In order to delineate the effect of the hydrostatic pressure on the retardation of the impactor and hence on deformations of the target panels, we have computed results with zero and nonzero values of these two parameters. We have exhibited in Fig. 13 the deformed shapes of the 4.45 -mm thick flat and positively curved panels $(R=127 \mathrm{~mm})$ as well as distributions of the effective plastic strain on a central cross section. By comparing results on the left side of the vertical dividing line to those on its right side, we conclude that the pressure dependence of the effective plastic strain at a point upon the hydrostatic pressure there has a negligible effect on the impact performance of the flat and the positively curved panels. As mentioned above, our earlier parameter sensitivity studies also indicated that plastic dissipation is essentially insensitive to these two parameters.

\section{Conclusions}

We have analyzed by the finite-element method the transient large deformations of clamped flat and curved polycarbonate (PC) panels of five different thicknesses impacted at normal incidence at the center by a $104 \mathrm{~g}$ hemispherical-nosed steel circular cylinder moving at different speeds. The PC has been modeled as thermoelastoviscoplastic, and the steel cylinder as smooth and rigid.

The mathematical model for low and high velocity impact has been validated by comparing the experimental and numerical deflections and the deformed shapes of PC plates for various thicknesses and impact speeds. By comparing the minimum impact speeds used in tests and numerical simulations for complete perforation of flat plates of three thicknesses, the effective plastic strain at failure was found to be 3.0. Subsequently, this value of the failure strain was used to characterize the effect of panel curvature and thickness on its impact resistance. It has been found that the negative curvature of the panel degrades its resistance to impact loading. The differences in impact responses for thin and thick panels found herein are summarized below.

- For thin plates, the positive curvature greatly enhances the impact resistance. In particular, we found that the impactor kinetic energies required for failure of the 3.00- and 4.45$\mathrm{mm}$ thick panels with $127 \mathrm{~mm}$ radius of positive curvature were three times those for their respective flat plates. It is primarily due to the formation of an elastic hinge around the impact point that results in subsequent bending deformations concentrated in this region till the panel bounces back.

- The positive curvature did not significantly improve the impact resistance of the thicker panels considered in this study. The overall impact resistance of those panels exhibited less sensitivity to the curvature than that of the thin panels.

- The hole is formed due to the deletion of failed elements rather than due to the ejection of a plug.

- The dependence of the effective strain rate at a point upon the hydrostatic pressure has very little effect on the retardation of the impactor and panel's deformations.

- The frictional force at the impactor/panel interface has negligible effect on the reaction force at the impactor/panel interface.
The improved impact resistance of thin panels with positive curvature is due to the decrease in the impacted region of (i) the average axial stresses, (ii) the average axial stretch, and (iii) the effective stress from that in the corresponding flat plates, as well as due to the development of an elastic hinge surrounding the impact point. However, for the thicker panels the positive curvature did not significantly decrease the effective stress near the plate center relative to that in flat plates and no hinge formed. The stretching deformations are dominant in thin panels and account for the difference in the impact response of thin and thick flat and curved panels.

\section{Acknowledgment}

This research was sponsored by the Army Research Laboratory and was accomplished under Cooperative Agreement No. W911NF-06-2-0014. The U.S. Government is authorized to reproduce and distribute reprints for Government purposes notwithstanding any copyright notation hereon.

\section{References}

[1] Radin, J., and Goldsmith, W., 1988, "Normal Projectile Penetration and Perforation of Layered Targets," Int. J. Impact Eng., 7(2), pp. 229-259.

[2] Sands, J., Patel, P., Dehmer, P., and Hsieh, A., 2004, "Protecting the Future Force: Transparent Materials Safeguard the Army's Vision," AMPTIAC Q., 8 , pp. 28-36.

[3] Siviour, C. R., Walley, S. M., Proud, W. G., and Field, J. E., 2005, “The High Strain Rate Compressive Behaviour of Polycarbonate and Polyvinylidene Difluoride," Polymer, 46(26), pp. 12546-12555.

[4] Moy, P., Weerasooriya, T., Hsieh, A., and Chen, W., 2003, "Strain Rate Response of a Polycarbonate Under Uniaxial Compression," SEM Conference on Experimental Mechanics, T. Proulx, ed., Society for Experimental Mechanics, Bethel, CT, pp. 2-4.

[5] Mulliken, A. D., and Boyce, M. C., 2006, "Mechanics of the Rate-Dependent Elastic-Plastic Deformation of Glassy Polymers From Low to High Strain Rates," Int. J. Solids Struct., 43(5), pp. 1331-1356.

[6] Mulliken, A. D., 2006, Mechanics of Amorphous Polymers and Polymer Nanocomposites During High Rate Deformation, Massachusetts Institute of Technology, Cambridge, MA, p. 290.

[7] Richeton, J., Ahzi, S., Daridon, L., and Remond, Y., 2005, "A Formulation of the Cooperative Model for the Yield Stress of Amorphous Polymers for a Wide Range of Strain Rates and Temperatures," Polymer, 46(16), pp. 6035-6043.

[8] Richeton, J., Schlatter, G., Vecchio, K. S., Remond, Y., and Ahzi, S., 2005, "A Unified Model for Stiffness Modulus of Amorphous Polymers Across Transition Temperatures and Strain Rates," Polymer, 46(19), pp. 8194-8201.

[9] Richeton, J., Ahzi, S., Vecchio, K. S., Jiang, F. C., and Adharapurapu, R. R., 2006, "Influence of Temperature and Strain Rate on the Mechanical Behavior of Three Amorphous Polymers: Characterization and Modeling of the Compressive Yield Stress,” Int. J. Solids Struct., 43(7-8), pp. 2318-2335.

[10] Fleck, N. A., Stronge, W. J., and Liu, J. H., 1990, "High Strain-Rate Shear Response of Polycarbonate and Polymethyl Methacrylate," Proc. R. Soc. London A, 429(1877), pp. 459-479.

[11] Ramakrishnan, K. R., 2009, "Low Velocity Impact Behaviour of Unreinforced Bi-Layer Plastic Laminates," Australian Defence Force Academy, Canberra, Australia.

[12] Rittel, D., 2000, "An Investigation of the Heat Generated During Cyclic Loading of Two Glassy Polymers_-Part I: Experimental," Mech. Mater., 32(3), pp. 131-147.

[13] Rittel, D., and Rabin, Y., 2000, "An Investigation of the Heat Generated During Cyclic Loading of Two Glassy Polymers-Part II: Thermal Analysis," Mech. Mater., 32(3), pp. 149-159.

[14] Rittel, D., 1999, "On the Conversion of Plastic Work to Heat During High Strain Rate Deformation of Glassy Polymers," Mech. Mater., 31(2), pp. 131-139.

[15] Richeton, J., Ahzi, S., Vecchio, K. S., Jiang, F. C., and Makradi, A., 2007, "Modeling and Validation of the Large Deformation Inelastic Response of Amorphous Polymers Over a Wide Range of Temperatures and Strain Rates," Int. J. Solids Struct., 44(24), pp. 7938-7954.

[16] Tervoort, T., Smit, R., Brekelmans, W., and Govaert, L. E., 1997, “A Constitutive Equation for the Elasto-Viscoplastic Deformation of Glassy Polymers," Mech. Time-Depend. Mater.," 1(3), pp. 269-291.

[17] Boyce, M. C., Parks, D. M., and Argon, A. S., 1988, "Large Inelastic Deformation of Glassy-Polymers-1: Rate Dependent Constitutive Model," Mech. Mater., 7(1), pp. 15-33.

[18] Varghese, A. G., and Batra, R. C., 2009, "Constitutive Equations for Thermomechanical Deformations of Glassy Polymers," Int. J. Solids Struct., 46(22-23), pp. 4079-4094.

[19] Varghese, A. G., and Batra, R. C., 2011, "Strain Localization in Polycarbonates Deformed at High Strain Rates," J. Polym. Eng., 31(6-7), pp. 495-519.

[20] Safari, K. H., Zamani, J., Ferreira, F. J., and Guedes, R. M., 2013, "Constitutive Modeling of Polycarbonate During High Strain Rate Deformation," Polym. Eng. Sci., 53(4), pp. 752-761. 
[21] Chang, F. C., and Chu, L. H., 1992, "Coexistence of Ductile, Semi-Ductile, and Brittle Fractures of Polycarbonate," J. Appl. Polym. Sci., 44(9), pp. 1615-1623.

[22] Mills, N., 1976, "The Mechanism of Brittle Fracture in Notched Impact Tests on Polycarbonate," J. Mater. Sci., 11(2), pp. 363-375.

[23] Fraser, R., and Ward, I., 1977, "The Impact Fracture Behaviour of Notched Specimens of Polycarbonate," J. Mater. Sci., 12(3), pp. 459-468.

[24] Allen, G., Morley, D., and Williams, T., 1973, "The Impact Strength of Polycarbonate," J. Mater. Sci., 8(10), pp. 1449-1452.

[25] Rittel, D., Levin, R., and Maigre, H., 1977, "On Dynamic Crack Initiation in Polycarbonate Under Mixed-Mode Loading," Mech. Res. Commun., 24(1), pp. 57-64.

[26] Plati, E., and Williams, J., 1975, "Effect of Temperature on the Impact Fracture Toughness of Polymers," Polymer, 16(12), pp. 915-920.

[27] Plati, E., and Williams, J., 1975, "The Determination of the Fracture Parameter for Polymers in Impact," Polym. Eng. Sci., 15(6), pp. 470-477.

[28] Adams, G. C., Bender, R. G., Crouch, B. A., and Williams, J. G., 1990, "Impact Fracture-Toughness Tests on Polymers," Polym. Eng. Sci., 30(4), pp. 241-248.

[29] Curran, D. R., Shockey, D. A., and Seaman, L., 1973, "Dynamic Fracture Criteria for a Polycarbonate," J. Appl. Phys., 44(9), pp. 4025-4038.

[30] Rittel, D., and Levin, R., 1998, "Mode-Mixity and Dynamic Failure Mode Transitions in Polycarbonate," Mech. Mater., 30(3), pp. 197-216.

[31] Gunnarsson, C. A., Weerasooriya, T., and Moy, P., 2011, "Impact Response of PC/PMMA Composites," Dynamic Behavior of Materials, Vol. 1, Springer, New York, pp. 195-209.

[32] Kelly, P. M., 2001, "Lightweight Transparent Armour Systems for Combat Eyewear," 19th International Symposium of Balllistics, Interlaken, Switzerland, pp. 7-11.

[33] Dorogoy, A., Rittel, D., and Brill, A., 2011, "Experimentation and Modeling of Inclined Ballistic Impact in Thick Polycarbonate Plates," Int. J. Impact Eng., 38(10), pp. 804-814.

[34] Shah, Q. H., and Abakr, Y. A., 2008, "Effect of Distance From the Support on the Penetration Mechanism of Clamped Circular Polycarbonate Armor Plates," Int. J. Impact Eng., 35(11), pp. 1244-1250.
[35] Shah, Q. H., 2009, "Impact Resistance of a Rectangular Polycarbonate Armor Plate Subjected to Single and Multiple Impacts," Int. J. Impact Eng., 36(9), pp. 1128-1135.

[36] Livingstone, I., Richards, M., and Clegg, R., 1999, "Numerical and Experimental Investigation of Ballistic Performance of Transparent Armour Systems," Lightweight Armour System Symposium (LASS), Shrivenham, UK.

[37] Richards, M., Clegg, R., and Howlett, S., 1999, "Ballistic Performance Assessment of Glass Laminates Through Experimental and Numerical Investigation," 18th International Symposium on Ballistics, pp. 1123-1130.

[38] Hazell, P. J., Roberson, C. J., and Moutinho, M., 2008, "The Design of Mosaic Armour: The Influence of Tile Size on Ballistic Performance," Mater. Design, 29(8), pp. 1497-1503.

[39] Antoine, G., and Batra, R., 2015, "Low Velocity Impact of Flat and Doubly Curved Polycarbonate Panels," ASME J. Appl. Mech., 82(4), p. 041003.

[40] Khalili, S. M. R., Soroush, M., Davar, A., and Rahmani, O., 2011, "Finite Element Modeling of Low-Velocity Impact on Laminated Composite Plates and Cylindrical Shells," Compos. Struct., 93(5), pp. 1363-1375.

[41] Gunnarsson, C. A., Weerasooriya, T., and Moy, P., 2008, "Measurement of Transient Full-Field, Out-of-Plane Back Surface Displacements of Polycarbonate During Impact," 11th International Congress and Exposition on Experimental and Applied Mechanics, pp. 1403-1413.

[42] Gunnarsson, C. A., Ziemski, B., Weerasooriya, T., and Moy, P., 2009, "Deformation and Failure of Polycarbonate During Impact as a Function of Thickness," International Congress and Exposition on Experimental Mechanics and Applied Mechanics, Society for Experimental Mechanics, Albuequerque, NM, June 1-4, Curran Assoc., Redhook, NY, pp. 1500-1511.

[43] Batra, R., and Peng, Z., 1996, "Development of Shear Bands During the Perforation of a Steel Plate," Comput. Mech., 17(5), pp. 326-334.

[44] Batra, R., and Chen, X., 1994, "Effect of Frictional Force and Nose Shape on Axisymmetric Deformations of a Thick Thermoviscoplastic Target," Acta Mech., 106(1), pp. 87-105.

[45] Antoine, G. O., and Batra, R. C., 2015, "Sensitivity Analysis of LowVelocity Impact Response of Laminated Plates,” Int. J. Impact Eng., 78(4), pp. 64-80. 